\title{
Implantable Cardiac Defibrillator Deactivation During End-of-Life Care in the COVID-19 Pandemic
}

\author{
Sarah E. Myers, MS and Gregory L. Eastwood, MD
}

People with implantable cardiac defibrillators (ICDs) who are nearing the end of life are at risk for arrhythmias, which activate the ICD and may cause unnecessary shocks and suffering. Because ICDs have enabled more patients to live longer, they often succumb to noncardiac diseases and may be cared for by primary care physicians. Despite published recommendations 10 years ago regarding the management of ICDs during the end of life, over half of patients with ICDs who are dying still have not been offered the choice of deactivation. The Coronavirus disease 2019 (COVID-19) pandemic has complicated this issue and the need to discuss it because of practices that separate patients from loved ones and that modify the usual interactions of patients with doctors and nurses. We offer the following recommendations: (1) the management of ICDs at the end-of-life needs to be understood by all physicians who care for patients with ICDs; (2) discussions about deactivating the ICD should occur while patients have decision-making capacity and are clinically stable, beginning at the time of ICD implantation, then periodically at follow-up appointments, and certainly when a change in the patient's clinical status warrants a reconsideration of the goals of care; and (3) clinicians should compensate for the impediments to communication with patients and families associated with the COVID-19 pandemic, which includes patient isolation and restrictive visitor policies, by using devices that permit visual communication to reexamine goals of care, including defibrillator deactivation, in patients with ICDs who are expected to die. (J Am Board Fam Med 2021;34:474-476.)

Keywords: Cardiac Arrhythmia, COVID-19, Defibrillators, End of Life Care, Implantable Cardiac Defibrillators (ICDs), Pandemics, Patient Care Planning, Primary Care Physicians

\section{Introduction}

People with implantable cardiac defibrillators (ICDs) who are nearing the end of life are at risk for arrhythmias and consequent unnecessary shocks and suffering because the ICDs will deliver a shock when the heart stops if they are not deactivated. Under these circumstances, the question may arise whether to deactivate the ICD. The Coronavirus disease 2019 (COVID-19) pandemic has complicated this question and the need to discuss it because of practices that separate patients from loved ones and that modify the usual interactions of patients with health professionals.

This article was externally peer reviewed.

Submitted 12 October 2020; revised 14 January 2021; accepted 19 January 2021.

From the Upstate Medical University, State University of New York (SEM, GLE).

Funding: None.

Conflict of interest: None.

Corresponding author: Gregory L. Eastwood, MD, University Professor, State University of New York, Center for Bioethics and Humanities, SUNY Upstate Medical University, 750 East Adams Street, Syracuse, NY 13210, Phone: 315-464-8454 (Email: eastwood@upstate.edu).
The number of patients with ICDs has increased, largely because of expanded indications for implantation. In addition, advances in medical therapy have improved cardiac function so that many patients with ICDs live longer. ${ }^{1}$ For these reasons, many more patients with ICDs succumb to noncardiac diseases and are likely to be cared for by noncardiologists. ${ }^{2}$

COVID-infected patients with ICDs have a high risk of dying and end-of-life considerations may arise quickly in such patients. Because of this, the possibility of deactivating the ICD during end-of-life care should be discussed well before the patient is dying, ideally during the months and years that they are under the care of a primary care physician. Regardless of who cares for the patient at the end of life, the patient's wishes regarding the deactivation of their device should be known.

\section{Effects of the COVID-19 Pandemic on ICD Deactivation Decisions}

The COVID-19 pandemic has made clear the need for advance care planning ${ }^{3}$ and has substantially 
complicated end-of-life discussions. How such discussions occur or whether they occur at all may influence the care and experience of the dying person. Restrictive visiting policies, patient isolation, and reduced face-to-face contact with physicians and nurses hamper these conversations. This pandemic has underscored the importance of having such discussions while patients have decision-making capacity, are clinically stable, and with family or others present.

\section{Effects of COVID-19 on the Heart}

COVID-19 is associated with cardiovascular complications, including acute myocardial injury, myocarditis, arrhythmias, cardiogenic shock, and cardiac arrest. ${ }^{4}$ Cardiovascular magnetic resonance imaging in patients who recently recovered from COVID infection showed cardiac involvement in $78 \%$ and ongoing myocardial inflammation in $60 \%$, independent of preexisting conditions and severity. ${ }^{5}$ Further, pre-existing heart disease is associated with an increased risk of inhospital death among patients hospitalized with COVID-19. ${ }^{6}$ Thus, because COVID-19 is associated with the development of new heart disease and aggravation of existing heart disease, COVID-19 infection likely increases the risk of cardiac death in patients with ICDs.

\section{Consensus Statements}

In 2010, both the Heart Rhythm Society and the European Heart Rhythm Association issued consensus statements regarding the management of defibrillators during the end of life. ${ }^{7,8}$ They were intended to familiarize clinicians with the principles of device deactivation, emphasize multidisciplinary communication, and reassure clinicians legally and ethically. The statements acknowledged the patient's legal and ethical right to request withdrawal of any medical treatment, regardless of terminal illness and of whether the withdrawal of such treatment leads to death. Further, a physician who withdraws ICD treatment in response to a patient's wishes should not be regarded as participating in physician-assisted suicide or euthanasia.

Subsequent reports suggested that the consensus statements may have had an insufficient effect on the frequency of device deactivation in end-of-life care. ${ }^{2,9}$ Over half of the patients with ICDs who are nearing the end of their lives still have not been offered the choice of deactivation. In addition, because patients with ICDs are living longer, ${ }^{1}$ many succumb to other diseases, and most patients with ICDs die on non-cardiology services. ${ }^{2}$ Perhaps physicians continue to have concerns or are simply uninformed about ICD deactivation since the guidelines were published in cardiology journals.

\section{Improving the Care of Patients with ICDs at the End of Life}

We offer the following recommendations:

1. The management of ICDs at the end of life needs to be understood by all physicians who care for patients with ICDs at the end of life.

2. Discussions about deactivating the ICD should occur while patients have decision-making capacity, are clinically stable, and with family or persons of the patient's choosing. ${ }^{10}$ The first discussion should occur at the time of ICD implantation, then periodically at follow-up appointments, and certainly when a change in the patient's clinical status warrants a reconsideration of the goals of care.

3. It is important to compensate for the impediments to communication with patients and families associated with the COVID pandemic, which include patient isolation, restrictive visitor policies, and requirements that medical personnel wear personal protective equipment that distances them from patients. Devices that permit visual communication should be used to aid conventional methods of communication to reexamine and refine goals of care, including defibrillator deactivation, in patients with ICDs who are expected to die.

To see this article online, please go to: http://jabfm.org/content/ 34/3/474.full.

\section{References}

1. Lebner A, Smith MJ. Deactivating implantable cardioverter-defibrillators (ICDs) in cancer patients: A scoping review of ethical considerations. J Pain Manage 2017;10:99-105.

2. Westerdahl AK, Magnsjo J, Frykman V. Deactivation of implantable defibrillators at end of life - Can we do better? Int J Cardiol 2019;291:57-62.

3. Block BL, Smith AK, Sudore RL. During COVID19 , outpatient advance care planning is imperative: We need all hands on deck. (Letter). J Am Geriatr Soc 2020;68:1395-7. 
4. Kang Y, Chen T, Mui D, et al. Cardiovascular manifestations and treatment considerations in covid19. Heart 2020;106:1132-20.

5. Puntmann VO, Carerj ML, Wieters I, et al. Outcomes of cardiovascular magnetic resonance imaging in patients recently recovered from coronavirus disease 2019 (COVID-19). JAMA Cardiol 2020;5:1265 Published online July 27, 2020. Available from: doi: 10.1001/jamacardio.2020.3557 Accessed September 15, 2020.

6. Mehra MR, Desai SS, Kuy SR, Henry TD, Patel AN. Cardiovascular disease, drug therapy, and mortality in Covid-19. N Engl J Med 2020;382: e102.

7. Lampert R, Hayes DL, Annas GJ, et al. Hospice and Palliative Nurses Association. HRS Expert Consensus Statement on the management of cardiovascular implantable electronic devices (CIEDs) in patients nearing end of life or requesting withdrawal of therapy. Heart Rhythm 2010;7:1008-26.

8. Padeletti L, Arnar DO, Boncinelli L, et al. Heart Rhythm Society. EHRA Expert Consensus Statement on the management of cardiovascular implantable electronic devices in patients nearing end of life or requesting withdrawal of therapy. Europace 2010;12:1480-9.

9. Stoevelaar R, Brinkman-Stoppelenburg A, Geert van Driel A, et al. Trends in time in the management of the implantable cardioverter defibrillator in the last phase of life: a retrospective study of medical records. Eur J Cardiovasc Nurs 2019;18:449-57.

10. Garlitski AC. Management of cardiac implantable electronic devices in patients receiving palliative care. Available from: https:/www.uptodate.com/contents/ management-of-cardiac-implantable-electronic-devicesin-patients-receiving-palliative-care. Updated Jan 24, 2020. Accessed April 22, 2021. 